\title{
DESAFÍOS DEL SISTEMA EDUCATIVO COSTARRICENSE: UN NUEVO PARADIGMA DE LA ADMINISTRACIÓN DE LA EDUCACIÓN
}

\author{
Guiselle María Garbanzo Vargas \\ Directora de la Escuela de Administración Educativa \\ de la Universidad de Costa Rica \\ San José, Costa Rica \\ Víctor Hugo Orozco Delgado \\ Docente de la Escuela de Administración Educativa \\ de la Universidad de Costa Rica \\ San José, Costa Rica
}

Recibido 30-X-2007 • Aceptado 13-XI-2007 • Corregido 20-II-2008

\begin{abstract}
Resumen: La autora y el autor realizan una contextualización teórico-práctica sobre la que actúa la educación, y desde ella el papel que asume la administración de la educación como disciplina responsable de la gestión de las organizaciones educativas. Se parte de los diferentes desafíos que presenta la educación en el sistema educativo costarricense y las posibles tendencias epistemológicas de la administración de la educación, como condición para responder a estos desafíos de manera acertada.
\end{abstract}

Palabras clave: Administración de la educación, educación, administración, organizaciones educativas, desafíos de la educación costarricense.

\section{Contextualización}

La administración de la educación como disciplina no se debe a sí misma, se debe a una sociedad en particular, a un modelo de sociedad, a sus necesidades, sueños y desafíos, para lo cual requiere acompañarse de un paradigma administrativo, en concordancia con las propuestas curriculares del enfoque educativo al que se adscribe.

La administración de la educación se enriquece a sí misma en la medida en que trabaje articulada al proyecto social. Al interactuar con la sociedad, se retroalimenta en doble vía, se nutre de los procesos sociales, económicos y espirituales de una nación determinada (Lucio, 2005).

Asumir una clara interpretación de los retos y desafíos a los cuales se enfrenta la educación, es una condición indispensable para la administración de la educación, para ello es necesario partir de un reconocimiento acertado e integral del escenario 


\begin{abstract}
The authors make a theoretical-practical context analysis in which education acts, and from it the role assumed by the Education Administration as the discipline responsible of the management of education organizations. It starts from the different challenges presented by education in the Costa Rican system and the potential epistemological trends of Education Administration, as a condition to respond to these challenges in an effective manner.
\end{abstract}

Key words: Education Administration, education, administration, education organizations, Costa Rican education challenges. bajo el cual actúa la educación. Este escenario es altamente complejo, desafiante y dinámico, debido a la constante interacción de diferentes variables de los sistemas socioeconómicos que intervienen en las políticas de desarrollo social, según parámetros de competitividad que la misma sociedad establece a escala mundial, lo que repercute en las diferentes realidades socioeducativas.

El actual contexto mundial trae implícito diferentes variables que, directa e indirectamente intervienen en el tipo de educación que se plasma en una sociedad determinada. La producción acelerada del conocimiento es una de estas variables y quizás la que más retos le ha puesto a los sistemas educativos en los últimos años. Hoy, más que nunca, el conocimiento, además de la capacidad que debe tener el individuo de adquirirlo, requiere también de la habilidad para su interpretación y aplicación responsable.

La presente coyuntura mundial ha exigido una revisión de los distintos sistemas educativos, con el fin de buscar una articulación pertinente con las demandas sociales. Ante lo cual, la educación se enfrenta con un reto impostergable, de evaluar, en forma propositiva, su rol ante la sociedad; este tipo de replanteamiento debe ocupar un lugar prioritario en las agendas de desarrollo social.

Son muchos los problemas que a lo largo de la historia ha enfrentado la educación; ante ellos, también son múltiples los esfuerzos al respecto, siendo quizás la principal preocupación acabar con los estados de miseria social que limitan el desarrollo de la educación en su sentido más amplio.

En América Latina y el Caribe, si bien es cierto se han logrado avances sustanciales en materia educativa, estos siguen siendo incompletos. Persisten serias lagunas en cuanto a la calidad, equidad y eficiencia del sistema educativo, que por el nivel de desarrollo a escala general, la región es colocada en el escenario del siglo 
XXI, sin embargo, hay deficiencias que "la mantienen anclada en el siglo XIX: tal es la paradoja educativa del subcontinente" (Rivero, 1999, p. 49).

La esperanza para alejar la miseria y la pobreza está en la educación principalmente; no obstante, no se debe caer en el juego conceptual de entender la educación institucionalizada como la única responsable de la adversidad; sin embargo, hay que tener en cuenta que la educación actúa en un sistema integral, nutrido por políticas estatales, respaldadas por enfoques económicos según la visión de quienes asumen las estructuras de poder en el contexto mundial, regional y nacional, donde subyacen distintos criterios de aplicación y, por supuesto, sustentada en un modelo de gestión educativa; aspectos que vienen a incidir en la sociedad como un todo.

Ante un panorama contextual ampliamente complejo, la educación hace uso de la administración para poder conducir todos los procesos educativos en procura de resultados educativos de calidad; es así como la administración en el campo de la educación asume un papel relevante, para armonizar todas las variables que intervienen en su proceso, tal y como describe Kast y Rosenweig en su teoría de la administración (2000, p. 21): "La función principal de la administración es desarrollar la congruencia entre los diferentes subsistemas y crear un clima que conduzca a la excelencia: efectividad, eficiencia y satisfacción del participante".

En este sentido la administración aplicada al campo de la educación conlleva la responsabilidad de conducir adecuadamente los procesos de gestión educativa en los distintos niveles y modalidades de la educación, articulada a un fuerte enfoque pedagógico, para poder alcanzar el desarrollo de los aprendizajes con altos parámetros de calidad. Para ello, es importante partir de que la educación es el resultado de diferentes interacciones que se producen en el contexto educativo, las que, a su vez, son determinadas por las políticas educativas que las instancias administrativas elaboran para producir las prácticas educativas, según las exigencias del escenario político y económico de cada época.

Para lograr los propósitos de la administración aplicada a la educación, se hace indispensable, entonces, comprender, en primera instancia, las dimensiones educativas sociales desde un enfoque analítico, crítico, reflexivo y propositivo, lo cual implica un esfuerzo, como lo expone Pérez (1999), por analizar los aspectos macro y micro entre las correspondencias y discrepancias incorporadas en las interacciones del contexto educativo, entendiendo que la educación conlleva un proceso amplio, flexible y dinámico, conducente a desarrollar, de manera equilibrada y equitativa, las capacidades de las personas, lo que es considerado por Lucio (2005) un derecho inalienable de todas las personas, para promover el desarrollo de todas las capacidades y conocimientos al servicio del desarrollo humano y económico de la sociedad.

El anterior contexto conduce a un replanteamiento sobre los desafíos que enfrenta la educación costarricense y el papel que debe asumir la administración de la educación, con el propósito de cerrar las brechas existentes en este campo, y hacer los cambios requeridos ante un mundo globalizado, donde la esperanza es un desarrollo sostenible con equidad, calidad y justicia social. No hay duda de que elevar la capacidad de gestión de las organizaciones educativas representa el principal reto para la administración de la educación.

\section{La educación costarricense y sus desafios}

Son muchos los desafíos que enfrenta la educación costarricense en la actualidad; muchos de estos han persistido a lo largo de la historia educativa en diferentes niveles de profundidad. El último Informe del Estado de la Educación ${ }^{1}$, publicado en el 2005 y fundamentado con datos del 2004, 
evidenció, ante la comunidad nacional, mediante una serie de macroindicadores, una serie de aspectos de interés nacional, que de una $u$ otra forma han persistido en la sociedad costarricense; estos resultados no han hecho otra cosa que desnudar al sistema educativo ante una serie de inconsistencias de las que hace rato se viene hablando. De manera que el panorama que se ofrece por medio del informe no es desconocido, y se traduce en los siguientes desafíos:

- universalizar la cobertura preescolar y secundaria.

- mejorar la calidad del sistema educativo,

- reducir la reprobación y mejorar la eficiencia,

- retener a los estudiantes y las estudiantes del sistema educativo,

- disminuir brechas socio-espaciales,

- ampliar la inversión en educación,

- mejorar las precarias condiciones de trabajo de las docentes y los docentes,

- mejorar la rectoría del sector,

- reforma institucional,

- fortalecer el sistema de monitoreo y evaluación,

- fortalecer la investigación educativa,

- reforzar el papel de las universidades públicas como agentes, de movilidad social,

- mejorar el monitoreo y el control de calidad de la enseñanza universitaria,

- fortalecer el vínculo entre la generación del conocimiento y su aplicación al desarrollo (Programa Estado de la Nación en Desarrollo Humano Sostenible, 2005) $)^{2}$.

Los desafíos expuestos expresan condiciones educativas que impactan a la sociedad costarricense, porque bajos resultados educativos conducen a:

- pocas posibilidades de movilidad social,
- $\quad$ aumento de la pobreza,

- pocas opciones para que los padres y madres mantengan sus hijos en las aulas, y

- $\quad$ efectos económicos significativos para la sociedad, entre otros.

La respuesta a todos estos desafíos parece representar una utopía por sí mismos para el sistema educativo por la complejidad implícita que conlleva; sin embargo, es posible minimizar las brechas existentes en cada uno de ellos en la medida en que sean abordados, en forma oportuna y responsable, por quienes poseen el compromiso social de dirigir la educación costarricense desde una perspectiva holística, en la que intervenga tanto la sociedad civil como las autoridades del Estado.

Precisamente, parte de esta responsabilidad la tienen todos aquellos profesionales en el campo de la administración de la educación, quienes, desde diferentes niveles y modalidades de la educación, asumen por su voluntad la decisión de conducir la educación hacia los mejores niveles de calidad; eso sí, no hay que perder de vista las condiciones contextuales según las cuales asumen el compromiso de gestar la educación, y dentro de estas condiciones intervienen tanto factores propios del sistema educativo como: presupuestarios, culturales, estructurales, concepciones filosóficas, así como la idoneidad profesional, entre otros.

\section{Administración de la educación}

El objeto de estudio de la administración de la educación recae en la gestión de la organización educativa, y las organizaciones educativas poseen diferentes características estructurales según niveles y modalidades de la educación.

Como disciplina, la administración de la educación se nutre de distintas áreas disciplinares: las ciencias de la educación, la teoría administrativa en 
general y disciplinas como la Psicología, la Sociología, la Economía, el Derecho y Ciencias Políticas, entre otras. (Salas, 2003). Todo este contexto disciplinar la hace poseedora de una posición epistemológica holística, lo que le da la capacidad de actuar en los variados entornos sociales a los que se somete la educación; entornos característicos por un sinfín de dificultades, como la deserción, la pobreza, la equidad, la infraestructura, y la preocupante exclusión educativa, entre otros. Estos desafíos exponen a la administración de la educación a su principal reto: a su capacidad de gestión en aras de la construcción de un desarrollo sostenible con equidad, calidad y justicia.

$\mathrm{Al}$ respecto, América Latina representa la región más desigual del orbe, con un 0,57 de acuerdo con el coeficiente de Gini (indicador que se emplea con frecuencia para medir la desigualdad; cuanto más se acerca a 1, peor es), cifra que se plasma en las siguientes estadísticas citadas por Kliksberg (2004, p. 26):

En el campo educativo, solo el $14 \%$ de los niños se halla en preescolar, el 50\% de los que ingresan en la escuela primaria desertan antes de completar el quinto grado, amplios sectores de jóvenes se hallan fuera de la secundaria. La educación de adultos es muy limitada. América Latina tiene actualmente solo 5,2 años de estudios cursados por habitante.

En este entorno, son muchos los que dudan del componente estratégico que la educación tiene para lograr que las mayorías que viven en condiciones de pobreza desarrollen capacidades para alcanzar mejores niveles de vida. Desde esta perspectiva, adquiere un amplio sentido la relevancia en cuanto a que las organizaciones educativas sean lideradas en forma eficiente y eficaz, como lo dice Valeirón (2006, p. 19) al referirse a la gran población que vive en condiciones de pobreza sobre la importancia de que "un niño estudie en una escuela ricamente gestionada, y como tal, capaz de desarrollar en él lo que su contexto social de pobreza le limita, y en ocasiones, impide". El mismo autor considera que no hay peor situación que estudiantes pobres accedan a escuelas pobremente gestionadas, pues las condiciones de pobreza se ven ampliamente incrementadas.

Un centro educativo bien gestionado, a pesar de la adversidad que lo pueda acompañar, es capaz de producir cambios significativos en el proceso de aprendizaje. Es en este marco que la administración de la educación se vuelve estratégica desde su posición ontológica y epistemológica para la búsqueda del logro de la eficacia de sus fines particulares en las organizaciones educativas y de la eficiencia, haciendo uso racional de los recursos existentes.

La administración de la educación eficaz y eficiente es el medio oportuno para posibilitar los cambios requeridos en materia educativa; de lo contrario, se estaría al frente de una gestión en la que subyacen acciones estériles, que no obedecen a ninguna política educativa ni a ningún control. La administración de la educación actúa sobre un paradigma específico contextual, político y estatal, que responde a la concreción del proyecto educativo.

En la administración de la educación recae la responsabilidad de liderar los cambios que el sistema educativo espera, en procura de las condiciones necesarias para incidir socialmente desde la educación. Opera en un determinado sistema educativo y está directamente vinculada con la visión epistemológica educativa y de desarrollo social al modelo que se adscriba. Debe estar atenta a responder a las demandas del entorno que se presentan en forma dinámica.

La administración de la educación, desde un enfoque de competitividad, debe estar en correspondencia con las necesidades de transformación social y económicas del país; para lo que se requiere de la articulación de estrategias y políticas educativas en conjunto con las políticas del Estado, en lo que se desea potenciar socialmente, a partir de un enfoque de equidad social; solo así podría el sistema educativo contribuir a producir el capital humano requerido. 
Para enfrentar los diferentes desafíos que interpelan a la educación, se requiere de un nuevo paradigma de la administración de la educación, epistemológicamente renovada, de manera que el sistema educativo trabaje de acuerdo con parámetros de calidad, como condición para aspirar a lograr, con mayor pertinencia, los fines $\mathrm{y}$ objetivos de la educación.

Antes de pasar a desarrollar las que se consideran tendencias de la administración de la educación ante los diferentes retos que esta enfrenta en la actualidad, debe quedar claro que no todos los problemas que golpean el sistema educativo son responsabilidad de los administradores y administradoras de la educación; problemas como la deserción, la repitencia y la exclusión, por ejemplo, apuntan a un problema sistémico derivado del contexto vulnerable en que actúa la educación, donde se plasman factores sociales, como el desempleo y la desintegración familiar por ejemplo, los cuales repercuten significativamente.

\section{Tendencias de la administración de la educación ante los desafios contextuales}

\subsection{Administración de la educación sustentada en una visión pedagógica}

Las coordenadas teórico-explicativas del componente curricular representan para la administración de la educación su punto de partida y final; es el centro de su gestión y desde ella posee la responsabilidad de propiciar los espacios para que el currículo se realice en forma eficaz y eficiente, propiciando coherencia entre los aprendizajes y los contextos sociales. A su vez, promueve procesos dinámicos curriculares adecuados a las exigencias del entorno, desarrolla la labor pedagógica apoyada en el monitoreo, evaluación y retroalimentación.
La administración del currículo conceptualmente posee una amplia complejidad explicativa, pues en ella intervienen distintas variables. La administración curricular se complementa de los principios teóricos de la administración y de la teoría educativa, cuya interacción genera los principios curriculares fundamentales dentro de los componentes del proceso educativo, de manera que la administración curricular es la función más importante que tiene la administración de la educación.

En la actualidad, hay conciencia de que para el logro de mejores niveles de calidad de la educación es fundamental fortalecer los procesos de gestión curricular en las distintas modalidades del sector educativo, mediante acompañamientos pedagógicos permanentes. La administración de la educación, sustentada en lo pedagógico, favorece espacios idóneos para la ejecución de las políticas educativas en forma acertada.

Asimismo a la administración de la educación le corresponde hacer efectivo el currículo en forma pertinente; para ello debe propiciar los aprendizajes y, a su vez, realizar todos los ajustes necesarios para las transformaciones curriculares que en el acto de la implementación se requieran. Por otra parte, la implementación curricular es determinada por la posición epistemológica del proyecto educativo al que se adscribe.

En este sentido, propiciar espacios pertinentes para que el proceso de enseñanza-aprendizaje se lleve de la mejor manera, es tarea impostergable y permanente de la administración de la educación; para ello, se requiere de modelos de gestión curricular eficientes, pues los de corte tradicional y parciales no han sido eficaces, ya que han producido indicadores insuficientes y tasas no alentadoras en los resultados educativos.

Los enfoques tradicionales de corte cuantitativo han producido una ausencia significativa de acciones eficientes en el abordaje de los resultados académicos. Para el caso de Costa Rica, y según el Programa Estado de la Nación en Desarrollo Humano 
Sostenible (2005), en el Informe del Estado de la Educación preocupa, sobre manera, la situación deficiente que presenta secundaria, donde el $20 \%$ de los estudiantes se gradúa sin repetir, además de que 9,4 años es el tiempo promedio para graduarse, casi el doble de los cinco establecidos. Definitivamente, estos son retos por resolver de acuerdo con una administración sustentada en lo pedagógico; sin embargo, por sí misma, la administración es insuficiente, puesto que la educación es responsabilidad de todos los actores sociales, pero sí puede marcar la diferencia.

La administración de la educación, centrada en una visión pedagógica, le permite alejarse de la administración de rutinas administrativas, en las que se enfrasca el gestor de la educación, lo que, a su vez, lo desvía de una gestión centrada en lo curricular; razón de ser de las organizaciones educativas, eso sí, es fundamental perder la visión simplista de lo educativo que está muy adherida en esta disciplina (Lucio, 2005).

Las organizaciones educativas no se deben dirigir como cualquier empresa, pues las organizaciones educativas difieren del mundo empresarial en lo sustantivo, en razón de ser organizaciones pedagógicas, con un proyecto de sociedad implícito.

\subsection{Administración de la educación sustentada en los principios de calidad, equidad, pertinencia y eficiencia}

La calidad de la educación conceptualmente es bastante ambigua, debido a las distintas variables disciplinares, temporales y epistemológicas que la acompañan. Existen tantas concepciones como visiones teóricas. Al respecto, Morillejo, Rebolloso, Pozo y Fernández (1999) consideran fundamental estimar, en lo que a calidad educativa se refiere, variables como: la relación entre los costos y beneficios obtenidos, pertinencia con los estándares preestablecidos, congruencia con el logro de objetivos, consecución de la excelencia, prestigio de la organización, satisfacción de la comunidad educativa. Seibold (2000), por su lado, destaca un elemento fundamental en la calidad educativa, que es la unidad integral que posee entre las distintas variables que interactúan entre sí.

A pesar de la variedad de concepciones con que se quiera valorar la calidad de la educación, la aspiración es una educación de calidad, equidad, pertinencia y eficiencia; sin embargo, llegar a esta aspiración implica superar distintos obstáculos que se traslapan en la consecución de este tipo de educación que muchas veces parece representar una utopía.

Una educación de calidad es la que logra que el estudiantado aprenda lo que deben aprender en el tiempo estimado y de la mejor manera, haciendo uso racional de los recursos de que dispone el sistema para la concreción del proyecto educativo. Toma importancia en este enunciado que lo que se aprenda sea relevante para las necesidades del contexto, aparte de que implique una transformación cualitativa.

En la medida en que exista capacidad de gestión en concordancia con las necesidades del sistema educativo, con un norte claro hacia los estándares a los que se quiere llegar, es posible una mayor aproximación a los requerimientos socioeducativos; en el entendido de que, quienes lideran los procesos educativos deben asumir una actitud responsable con la labor encomendada, de manera que la calidad educativa que se propone alcanzar la administración sea la aspiración compartida por los actores involucrados, donde la comunidad educativa tenga claros los parámetros de calidad, pertinencia, eficiencia y eficacia que se desean lograr. La calidad de la educación es una responsabilidad compartida, donde los gestores de la educación deben tener la capacidad de transmitirla y concebirla, no solo como un producto, sino como un proceso por el que se lucha. 
Con respecto a la equidad, está claro que la educación es una responsabilidad de Estado; no obstante, una educación de calidad debería acompañarse de políticas que la promuevan; entendiendo esto como las condiciones que garantizan la máxima homogeneidad educativa entre los individuos, puesto que los estudiantes no poseen las mismas posibilidades educativas se da ausencia de equidad educativa. En este entorno, la administración de la educación promueve la equidad en la medida en que propicie la captación y distribución racional de los recursos con que cuenta, incluyendo los humanos, financieros $\mathrm{y}$ pedagógicos, para la concreción de la política educativa, en el entendido de que se requiere de una capacidad de gestión de alto nivel.

\subsection{Administración de la educación sustentada en una visión integradora}

La administración de la educación, en el cumplimiento de sus principios disciplinares, debe partir de un núcleo integrador, donde se visualicen todos los ámbitos de la educación con un enfoque holístico; concepción fundamental para propiciar procesos de gestión de calidad en los diferentes niveles y modalidades de las organizaciones educativas. La visión integradora parte de un núcleo generador que le da sustento a la educación hasta los núcleos medios, de las organizaciones educativas, hasta llegar al aula (Valeirón, 2006).

El núcleo generador incluye la comprensión del entorno donde actúa el sistema educativo; esto es, asumir una visión holística de las diferentes demandas contextuales a escala nacional, regional y global, desde las cuales el sistema educativo articula y ajusta sus políticas.

El núcleo medio se refiere a las diferentes direcciones y unidades de gestión que el sistema educativo requiere para operacionalizar el proyecto educativo, desde la posición más alta jerárquicamente hasta llegar a los medios operativos.

El núcleo de las organizaciones educativas involucra a todos los responsables de las organizaciones educativas, según los diferentes niveles de la educación, como la secundaria, primaria y preescolar. En este núcleo, la familia, las asociaciones comunales, las municipalidades, la Iglesia, y el sector privado, asumen un papel estratégico para la administración de la educación, pues la gestión educativa de calidad es responsabilidad de los actores sociales involucrados en la educación, lo cual incluye la sociedad civil en su totalidad.

Un reto de la administración de la educación es articular los diferentes subsistemas que intervienen en la gestión educativa, de manera que los resultados se obtengan con eficiencia, según los intereses sociales.

El último núcleo tiene lugar en el aula, donde se da la intervención del docente con la interacción del estudiantado y toma lugar el proceso de enseñanza-aprendizaje.

Una concepción de administración de la educación implica un posicionamiento epistemológico de los distintos niveles de la gestión. Una visión centrada en un solo núcleo carece de integridad conceptual y operativa, lo cual provoca conducciones organizacionales centradas en una visión limitada del marco de acción de la educación; por ende, sus resultados reflejan la posición que asuma el directivo.

La visión integral de la administración de la educación ante sus organizaciones es una condición indispensable para propiciar procesos de transformación que promuevan a su vez una educación de mayor calidad. Esta visión integradora comprende, además de la visión de gestión, las relaciones que se producen en la organización como un todo ante los objetivos por alcanzar. 


\subsection{Administración de la educación sustentada en una gestión flexible, dinámica y competitiva}

Las organizaciones educativas como todos los sistemas sociales son sistemas afectados por los diferentes cambios que se gestan en el ambiente; cambios que traen variables desconocidas e impredecibles; estas variables le dan el carácter complejo que acompaña a las organizaciones como un todo.

Al desempeñarse las organizaciones educativas sobre un sistema social, que por su propia naturaleza es complejo, donde distintos elementos interactúan entre sí, en forma independiente pero interrelacionada, y que como producto de esta relación se da una totalidad funcional, no podría comprenderse las organizaciones educativas más que la suma de la interacción de los subsistemas involucrados en ellas, es así que se requiere una gran capacidad de gestión flexible, dinámica y competitiva, lo anterior como condición estratégica para propiciar los cambios que la educación demanda.

Es por lo anterior que cobra relevancia la necesidad de superar los enfoques tradicionales de la administración de corte burocrático e inflexible. Este modelo ha limitado los espacios de acción de la educación, repercutiendo en áreas tan vulnerables como la equidad social.

La prevalencia de modelos de gestión excesivamente centralistas han representado un obstáculo en la dinámica educativa, impidiendo así el desarrollo de importantes programas y proyectos educativos. Sistemas de esta índole son limitantes para el proceso de planificación, seguimiento y evaluación de la gestión educativa, pues quedan en el camino propósitos sin concluir en razón de una serie de obstáculos propios de una gestión de corte burocrático y centralista (Lucio, 2005).

Las demandas contextuales de hoy exigen de la administración de la educación alejarse de una gestión tradicional, para asumir un papel protagónico y pertinente en relación con los desafíos que se le presentan; para ello, es necesario actuar en forma dinámica, flexible y competitiva, de manera que logre tener la capacidad de ajustarse a las demandas del entorno con responsabilidad gerencial.

A lo largo de la presente época Lucio (2005) considera que los sistemas educativos están transitando por un largo proceso de reconversión, que trae implícito el mejoramiento en la eficiencia de las organizaciones educativas, alejándose de patologías que mucho perjuicio le han causado en el pasado como:

- Modelos burocratizados.

- Gestión educativa basada en el anonimato.

- Superposición de tareas.

- Procesos administrativos lentos, ineficientes.

- $\quad$ Pérdida de sentido.

- Pérdida de calidad.

- $\quad$ Frustración personal y profesional.

Para combatir estas patologías, se hace imprescindible replantear la gestión educativa, orientada a:

- Asumir al ser humano como sujeto gestor de cambios.

- $\quad$ Propiciar la toma de decisiones participativa con responsabilidad.

- Mayor capacidad de negociación.

- Sustentar su accionar en la planificación y evaluación como medio estratégico para la sostenibilidad de las políticas educativas.

- Eficiencia y eficacia organizativa y administrativa de los recursos disponibles.

- $\quad$ Gestión basada en el liderazgo legítimo.

- Capacidad para trabajar en equipos y alejarse de la individualidad.

Este replanteamiento epistemológico debe concebirse en el marco de las organizaciones educativas, entendiéndolas como 
sistemas creados por el hombre, como lo expone Chiavenato (2007), que mantienen una dinámica interacción con el ambiente; estas organizaciones, a su vez influyen sobre el medio y este sobre ellas. Es así cómo las organizaciones educativas, al estar integradas por diferentes unidades sociales relacionadas entre sí, que le exigen diferentes demandas, no puede postergar un replanteamiento de su funcionalidad para cumplir con la responsabilidad social que tienen, en cuanto a alcanzar una educación de calidad, según los propósitos educativos adscritos a un proyecto social determinado.

\subsection{Administración de la educación sustentada en el desarrollo de una nueva cultura organizacional}

La cultura organizacional es un sistema de valores y creencias compartidas por los miembros de las organizaciones; se manifiesta en constante interacción con los individuos y la estructura organizacional. Es fuertemente influido por el estilo de administración prevaleciente, y se afecta por la naturaleza de la organización. La cultura organizacional se ve rodeada por una serie de variables que la determinan, como los valores individuales y organizacionales, normas y patrones de comportamiento. La interacción de estas variables, en conjunto con el estilo de administración, produce una determinada cultura organizacional.

En este contexto, el principal reto de la administración es alinear su organización hacia la misión y visión establecida, para lograr un involucramiento organizacional inteligente, en busca de manifestaciones de la cultura organizacional de manera flexible, innovadora, dinámica, oportuna a las exigencias del entorno y con un alto nivel de compromiso de las personas que la componen hacia el logro de los objetivos predeterminados.
En criterio de Sanzberro (2007), existen una serie de variables que hacen exitosa una cultura organizacional, entre ellas:

- fortaleza ante la debilidad de la cultura,

- $\quad$ identidad de los miembros,

- $\quad$ énfasis en el trabajo en equipo,

- $\quad$ énfasis en las personas, en las relaciones,

- $\quad$ autonomía organizacional,

- tolerancia del riesgo,

- $\quad$ actitud preactiva,

- la diversidad como valor organizacional.

Es compromiso de quienes lideran las organizaciones educativas realizar lo necesario para replantear el cambio de la cultura organizacional requerido, en torno a estas variables para cumplir la razón de ser de la organización educativa, independientemente de sus niveles y modalidades de la educación. Una adecuada dirección reestructura las conciencias colectivas, actitudes, conocimientos y prácticas, hacia la consecución exitosa de los objetivos educativos, de manera que la organización sea capaz de generar los cambios precisos en los integrantes de la organización (Valeirón, 2006).

Es responsabilidad de quienes asumen la conducción de la educación, desarrollar capacidad para modificar el paradigma dominante, a fin de crear una cultura organizacional, consecuente con los propósitos organizacionales, construyendo de esta manera una organización capaz de favorecer los cambios requeridos tanto en los individuos que la componen como en el resultado académico. El replanteamiento de la cultura organizacional depende de un liderazgo transformador, basado en una visión de futuro que se complementa con el conocimiento de la realidad social y los propósitos de la educación en ella. Debe implicar un aprendizaje e innovación constante y una mejora continua e innovadora. 


\subsection{Administración de la educación basada en la negociación como estrategia administrativa}

La manera como la dirección promueve y realiza su administración, determina el funcionamiento del proyecto educativo, el cual supone un compromiso institucional consensuado por parte de las personas que lo asumen (Gairín, 2007).

La administración que renuncia a la negociación decide el camino rígido e inflexible de la administración; un enfoque nada alentador para las organizaciones. Para que la negociación sea asumida como una política de la administración, requiere de un alto grado de madurez emocional y profesional de quien lidera la organización educativa.

Una política basada en la negociación propicia espacios para el diálogo, respeto, promoción de valores moralmente aceptados, sustentados en principios democráticos, de cuya interacción, además de favorecer una cultura organizacional inteligente, promueve un clima organizacional adecuado para el cumplimiento del deber ser.

La participación democrática promueve la construcción de un proyecto organizacional comprometido por las partes involucradas, incluyendo en el campo de la educación a la comunidad estudiantil, el profesorado y comunidad en general.

Los principios democráticos son una característica del Estado moderno, y como tales, deben subyacer en los modelos de gestión de calidad, cuando es a esta a la que se quiere aspirar. La gestión democrática favorece la corresponsabilidad, donde se facilita el diseño, ejecución, sostenibilidad y evaluación de los proyectos educativos.

Una administración basada en la participación se sustenta en el diálogo, con espacios paralelos, donde los diferentes actores sean partícipes del proyecto educativo organizacional. Estas condiciones son requisitos para implementar una cultura organizacional de consenso, alejada de la mera legalidad, que pocas ventajas le trae a la administración. La aplicación de procedimientos legalistas se da cuando la dirección ha renunciado a la negociación como principal estrategia administrativa.

\subsection{Administración de la educación con perspectiva humanista}

Es de rigor comprender e interiorizar, de parte de quienes gestan la educación que para alcanzar lo propuesto en los fines y políticas de la educación, conducentes a la formación del ser humano, que este no es solamente un ser racional, como lo expone Valeirón (2006); es, al mismo tiempo, físico, social, espiritual, histórico, biológico y psíquico, que vive en el mundo como un todo.

Boff (2007, p. 3), destacado humanista a nivel mundial considera que "la cooperación, la solidaridad, la interdependencia de unos y otros no es una ley entre otras; es la ley fundamental del universo y de la vida humana". Esta integración es la visión humanista que se requiere forjar en las organizaciones educativas para el sano cumplimiento de la operacionalización de los fines y políticas de la educación.

Últimamente, en los debates teóricos sobre la cultura organizacional, se reconoce que una de las razones que hace exitosa una organización es el alto valor asignado a las personas. Considera Sanzberro (2007), desde este punto de vista la administración se está volviendo humanista, al valorar al ser humano como el capital más valioso dentro de una organización. La administración debe orientarse hacia las relaciones personales, sin descuidar las tareas.

El fin último de la educación es ofrecerle a la sociedad individuos competitivos, profesional y personalmente, cuya formación debe ser regida por la cooperación y no por la competencia que lideren las transformaciones sociales que el contexto demanda. Asumir una perspectiva de desarrollo humano debe ser una prioridad para 
la administración de la educación, centrada en los más nobles valores sociales, en conjunto con el proceso de formación.

\subsection{Administración sostenible y planificada}

Las políticas educativas requieren para su eficiente operacionalización de profesionales en el campo de la administración con habilidades gerenciales, para darles sostenibilidad. Lo que implica procesos de formulación, ejecución, monitoreo, evaluación y retroalimentación.

Una administración acertada de la educación requiere la capacidad de los directivos para darle sostenibilidad a los diferentes proyectos que se desarrollan en sus organizaciones educativas, sustentada en los principios de la planificación. Además, es fundamental el control administrativo de las distintas acciones inherentes a los diferentes proyectos, con el propósito de detectar, oportunamente, debilidades para lo cual la evaluación dentro del proceso de la planificación es una herramienta imprescindible.

Una administración cuyos fundamentos se basan en la planificación, se caracteriza por concederle importancia a los resultados educativos (Lucio, 2005). Los gestores de la educación requieren elaborar estrategias de intervención apoyados en procesos de planificación que les permitan diseñar y desarrollar diferentes proyectos para que contribuyan, desde su gestión y espacios de acción, a dar respuestas oportunas a las demandas inherentes de la educación, sin perder de vista que la educación es responsabilidad de todos. No es un problema exclusivo del Ministerio de Educación, ni de los políticos, sino de todos los involucrados en distintos niveles de responsabilidad del sistema educativo.

Como parte del proceso de planificación aplicado en el campo educativo en aras de la consecución de sus políticas, la administración de la educación, siendo consecuente con su propia naturaleza, necesita del acompañamiento de la evaluación sistemática aplicada en todos los niveles y modalidades de la educación. La evaluación permite monitorear su accionar, según los estándares preestablecidos hacia la consecución de la misión organizacional.

Seguidamente, se ilustran las tendencias de la administración de la educación desarrolladas anteriormente, con el propósito de ofrecer una mayor comprensión explicativa:

Figura $\mathrm{N}^{\mathrm{o}} 1$

Tendencias de la administración de la educación ante los desafíos contextuales

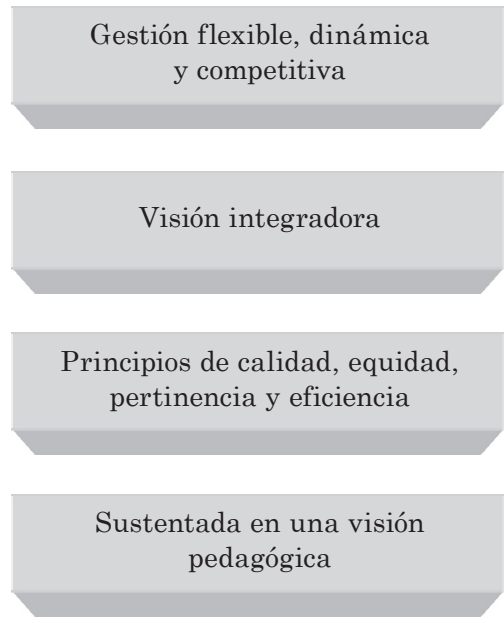

Fuente: Garbanzo y Orozco, 2007.



Desarrollo de una nueva


administrativa

Perspectiva humanista

Sostenible y planificada 
Ahora bien, para que la administración de la educación se acerque en forma significativa a las anteriores tendencias de la administración planteadas, se requiere de una serie de condiciones fundamentales, dentro de las organizaciones educativas, que posibiliten su marco de acción. A continuación se presentarán algunas de las más sustanciales.

\section{Condiciones organizacionales en la administración de la educación}

\subsection{Autonomía organizacional}

La autonomía de los centros educativos es la facultad de tomar decisiones propias sobre la organización y desarrollo de la actividad educativa; dicho planteamiento ha ocupado una constante presente en el debate pedagógico de las últimas décadas (Gairín, 2007).

Las organizaciones educativas, en sus distintos niveles de acción, para propiciar una eficiente administración de la educación requieren de mayores niveles de autonomía y descentralización de sus recursos, para lo que se hace necesario mayor independencia en la toma de decisiones; siendo esta un elemento clave para los gerentes (Chiavenato, 2007). La autonomía como herramienta de gestión de los administradores y las administradoras de la educación es una condición importante para transferir poder de decisión local y sentar responsabilidades en quienes administran.

La autonomía organizacional permite al directivo de la educación empoderarse, dirigir con sentido de responsabilidad la conducción de la educación que decidió asumir en forma voluntaria, respondiendo por sus resultados y las consecuencias de sus decisiones; nadie más que él, es quien debe responder. Es una particularidad de la administración de la educación encauzar, en forma comprometida, a los miembros de la organización hacia los objetivos planteados; esta conducción, desde un enfoque de autonomía, implica asumir las consecuencias de las acciones tomadas y empoderar la organización ante los resultados.

\subsection{Articulación de la formación del recurso humano con las universidades responsables}

La coordinación y la articulación entre el sistema educativo y las universidades formadoras de sus profesionales, representan un desafío valioso por considerar por parte de la administración de la educación, y a su vez una condición necesaria para alcanzar mejores resultados en materia educativa.

Las universidades y el sistema educativo deben coordinar entre sí la planificación de la formación de sus recursos humanos, sustentada en los requerimientos del desarrollo social. No asumir una política integrada en esta materia genera importantes vacíos para la gestión educativa en la consecución de sus objetivos a nivel general.

El fraccionamiento y la desarticulación entre el Ministerio de Educación y el sector terciario de la educación, lejos de ser una condición que debe operar, es una posición que le resulta sumamente costosa al Estado. Ante este vacío, se hace cada día más evidente la necesidad de contar con el nivel adecuado en formación y capacitación profesional, para generar programas de desarrollo profesional, y de formación académica coherentes con los paradigmas de desarrollo social requeridos (Lucio, 2005).

La formación profesional y los espacios de programas de capacitación son trascendentales para aspirar a un alto nivel de calidad educativa desde la administración de la educación. Una eficiente administración está articulada con la calidad del recurso humano con que cuenta, para lograr la consecución de los fines de la educación; de lo contrario, se cae en un 
derroche de esfuerzos sin sostenibilidad que no aporta beneficio social alguno. En esta materia, hay que reconocer importantes esfuerzos que en los últimos años ha hecho el Ministerio de Educación Pública en programas de capacitación, principalmente en conjunto con las universidades públicas.

Se requiere una reorientación profesional con responsabilidad social, basada en un perfil profesional de corte reflexivo, crítico, propositivo actualizado, dinámico y comprometido con el proyecto educativo.

No se puede esperar que la administración de la educación resuelva gran parte de los problemas que acechan a la educación, si no se invierten esfuerzos en la profesionalización en forma articulada, por parte de las autoridades educativas responsables; esto es una condición impostergable para poder colocar a la educación en un escenario competitivo. Se pueden resolver problemas de corte administrativo, que también son importantes, pero si el problema de idoneidad del personal no se resuelve, difícilmente se llega a un cambio esperado.

\subsection{Currículos educativos pertinentes}

El currículo es un proceso englobante de los objetivos humanos, políticas sociales, culturales y económicas, encargados a la educación; supone una compleja interacción de actores y elementos que deben ser administrados. No obstante, la administración curricular supone actos de gestión, orientados a la planificación y control para el logro de los objetivos curriculares.

El currículo, para cumplir su función, debe ser coherente con las necesidades del desarrollo social y acompañarse de metodologías didácticas pertinentes con lo que se desea transmitir, y así como poseer capacidad para ajustarse a las necesidades del contexto según las demandas.

Es condición para contar con un currículo que responda a las necesidades de desarrollo social y económico del país, que prevalezca en primera instancia una política de Estado, con una misión y visión claras sobre el horizonte hacia el que se desea caminar, en busca de la potencialización de los ciudadanos, en un proyecto social acorde con las necesidades del momento. Estas políticas deben ser insertadas en políticas educativas para finalmente buscar su operacionalización. No contar con esta condición trae como consecuencia un desperdicio inevitable de recursos económicos, con repercusiones sociales imaginables; un lujo que ningún sistema educativo puede darse.

Revisar la pertinencia del currículo es tarea constante; para ello es fundamental posicionarse en una visión que propicie la creatividad, la criticidad, la búsqueda del conocimiento y la innovación, alejándose de paradigmas fragmentados y desarticulados, de corte memorístico, que mutilan la creatividad y la innovación; así lo deja ver el Programa Estado de la Nación en Desarrollo Humano Sostenible (2005), en su Informe del Estado de la Educación, al considerar que la filosofía de la educación y de la pedagogía de corte tradicionalista ya no son pertinentes con las nuevas demandas y exigencias sociales.

Los planteamientos curriculares y la gestión de la enseñanza y el aprendizaje deben encauzarse en forma vinculada y no dejarse guiar por intereses internos de la educación desarticulados con las demandas del país (Lucio, 2005). Es esencial que la administración de la educación asuma una visión de nación con perspectiva holística, integral, sistémica en concordancia con el sentir del proyecto social.

A continuación se ilustran las principales condiciones organizacionales que deben operar para que las tendencias de la administración educativa, propuestas en el presente artículo, se lleven a cabo de la forma más significativa. 
Figura $N^{\circ} 2$

Condiciones organizacionales en la administración de la educación

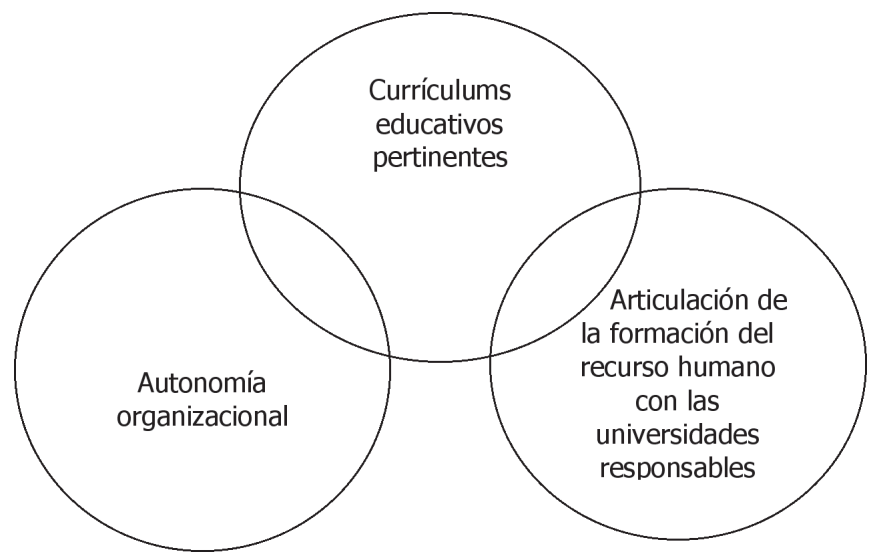

Fuente: Garbanzo y Orozco, 2007.

\section{Consideraciones finales}

La administración de la educación debe actuar articulada con las políticas públicas y hacer de la educación el eje dinamizador del desarrollo social al que se aspira. La administración de la educación está llamada a hacer que se operacionalicen las políticas educativas; para ello, requiere movilizar los recursos necesarios.

La articulación del proceso de enseñanza-aprendizaje con los planes de desarrollo del país, implica una relación impostergable y coherente con las políticas educativas y las públicas. No actuar de esta forma, considera (Lucio, 2005, p. 8), es propiciar una "gestión educativa autosuficiente, fragmentada y aislada del quehacer nacional, diluida y con poco impacto y sostenibilidad". Lo que explica la gran brecha entre el rezago de la educación en concordancia con las necesidades y expectativas sociales.

La respuesta a los diferentes desafíos que enfrenta la educación en el contexto de la posmodernidad es una labor de conjunto, no es una responsabilidad absoluta de la administración de la educación, es un compromiso articulado de todos, y por ser la educación un bien público, requiere un mayor esfuerzo del Estado, por parte de las autoridades correspondientes.

Indudablemente, en este entorno los profesionales en el campo de la administración de la educación poseen una doble responsabilidad en cuanto a los resultados de sus gestiones; en primera instancia, se decide administrar la educación en forma voluntaria, acción que implica, por sí misma, una alta responsabilidad social que no se le impuso.

En segundo lugar, los administradores y administradoras de la educación que asumen el reto de dirigir la educación, indistintamente del nivel educativo en que se inserten, son los responsables de los resultados de sus organizaciones educativas, sin obviar las limitaciones que el entorno ofrece. Precisamente porque es complejo el medio sobre el cual actúa la educación, toma relevancia el posicionamiento de la administración de la educación como disciplina que, ontológica y epistemológicamente se debe a la gestión de las organizaciones educativas.

El actual entorno social, caracterizado por la sociedad del conocimiento, impone a estos profesionales la necesidad de desarrollar habilidades para trabajar en ambientes altamente complejos, así como la capacidad de una adecuada comunicación, 
de delegación, de una actitud positiva hacia el aprendizaje y la innovación, y la impostergable intervención sistémica estratégica ante las múltiples demandas.

\section{Nota}

1. La investigación realizada surge por solicitud hecha por el entonces presidente de la República de Costa Rica, Dr. Abel Pacheco en el 2003, al Consejo Nacional de Rectores (CONARE), el cual fue creado mediante convenio para coordinar la educación superior pública universitaria.

2. CONARE, 2005. Los datos arrojados en el Informe del Estado de la Eduación, se sustenta de información de 2004.

\section{Referencias bibliográficas}

Boff, L.. (2007, marzo). Humanidades hoy en América Latina. Conferencia Inaugural dictada en la Universidad de Costa Rica (UCR). San José, Costa Rica.

Chiavenato, I. (2007). Introducción a la teoría general de la administración. Bogotá: McGraw-Hill.

Morillejo, E. A., Rebolloso P., E., Pozo, C. y Fernández, B. (1999, Noviembre). Evaluación y calidad en la educación superior. Papeles del Psicólogo, 74, 1-6.

Gairín, J. (2007, Mayo). Nuevos retos y perspectivas en la administración y gestión de la educación. Revista Electrónica de Gestión Educativa, 1, Artículo 14, 1-40. Extraído el 22 diciembre, 2006 de

http://www.eae.ucr.ac.cr/Revista/ Revista.html

Kast, F. y Rosenzweig, J. (2000). Administración en las Organizaciones ( $2^{\mathrm{a}}$ ed.). USA: McGraw-Hill.

Kliksberg, B. (2004). Hacia una Economía con Rostro Humano (9 ed.). San
José, Costa Rica: Instituto de Altos Estudios en Gerencia Social.

Lucio G., R. (2005, Noviembre). La Gestión Educativa: Una perspectiva innovadora y de largo aliento. Ponencia presentada en el II Congreso Internacional de Administradores de la Educación: Modelos de Gestión Educativa en la Sociedad Contemporánea porque el mundo ha cambiado, San José, Costa Rica: Universidad de Costa Rica.

Pérez G., Ángel I. (1999). La cultura escolar en la sociedad neoliberal. Madrid: Ediciones Morata, S. L.

Programa Estado de la Nación en Desarrollo Humano Sostenible. (2005). CONARE [Coord.]. Informe Estado de la Educación Costarricense (1). San José, Costa Rica: Programa del Estado de la Nación.

Rivero, J. (1999). Educación y Exclusión en América Latina. Reformas en tiempos de globalización. Lima, Perú: Tarea.

Salas M., F. E. (2003). La Administración Educativa y su fundamentación epistemológica. Revista Educación 1(27), 9-16.

Sanzberro, S. (2007). Curso Superior de Gestión y Liderazgo en Administración Universitaria. Universidad de Costa Rica. San José, Costa Rica.

Seibold, J. R. (2000, Mayo-Agosto). La calidad integral en educación. Reflexiones sobre un nuevo concepto de calidad educativa que integre valores y equidad educativa. Revista Iberoamericana de Educación, 23, 215-231.

Valeirón, J. (2006). Modelo de gestión de la calidad para los centros educativos. República Dominicana: Ed. Educativa. 